\title{
Aerosolized antibiotics for ventilator- associated pneumonia: a pairwise and Bayesian network meta-analysis
}

\author{
Feng Xu' ${ }^{1}$ Lu-Lu He${ }^{1}$, Luan-Qing Che ${ }^{1}$, Wen Li', Song-Min Ying ${ }^{1}$, Zhi-Hua Chen ${ }^{1}$ and Hua-Hao Shen ${ }^{1,2^{*}}$
}

\begin{abstract}
Background: Aerosolized antibiotics have been proposed as a novel and promising treatment option for the treatment of ventilator-associated pneumonia (VAP). However, the optimum aerosolized antibiotics for VAP remain uncertain.

Methods: We included studies from two systematic reviews and searched PubMed, EMBASE, and Cochrane databases for other studies. Eligible studies included randomized controlled trials and observational studies. Extracted data were analyzed by pairwise and network meta-analysis.

Results: Eight observational and eight randomized studies were identified for this analysis. By pairwise meta-analysis using intravenous antibiotics as the reference, patients treated with aerosolized antibiotics were associated with significantly higher rates of clinical recovery (risk ratio (RR) 1.21, 95\% confidence interval $(\mathrm{Cl}) 1.09-1.34 ; P=0.001$ ) and microbiological eradication (RR 1.42,95\% Cl 1.22-1.650; $P<0.0001$ ). There were no significant differences in the risks of mortality (RR 0.88, 95\% Cl 0.74-1.04; $P=0.127$ ) or nephrotoxicity (RR 1.00, 95\% Cl 0.72-1.39; $P=0.995$ ). Using network meta-analysis, clinical recovery benefits were seen only with aerosolized tobramycin and colistin (especially tobramycin), and microbiological eradication benefits were seen only with colistin. Aerosolized tobramycin was also associated with significantly lower mortality when compared with aerosolized amikacin and colistin and intravenous antibiotics. The assessment of rank probabilities indicated aerosolized tobramycin presented the greatest likelihood of having benefits for clinical recovery and mortality, and aerosolized colistin presented the best benefits for microbiological eradication.

Conclusions: Aerosolized antibiotics appear to be a useful treatment for VAP with respect to clinical recovery and microbiological eradication, and do not increase mortality or nephrotoxicity risks. Our network meta-analysis in patients with VAP suggests that clinical recovery benefits are associated with aerosolized tobramycin and colistin (especially tobramycin), microbiological eradication with aerosolized colistin, and survival with aerosolized tobramycin, mostly based on observational studies. Due to the low levels of evidence, definitive recommendations cannot be made before additional, large randomized studies are carried out.
\end{abstract}

Keywords: Aerosolized antibiotics, Ventilator-associated pneumonia, Multidrug-resistant pathogens, Meta-analysis, Network meta-analysis

\footnotetext{
* Correspondence: huahaoshen@zju.edu.cn

${ }^{1}$ Key Laboratory of Respiratory Disease of Zhejiang Province, Department of

Respiratory and Critical Care Medicine, The Second Affiliated Hospital,

Zhejiang University School of Medicine, Hangzhou, China

${ }^{2}$ State Key Laboratory of Respiratory Disease, Guangzhou, China
}

(c) The Author(s). 2018 Open Access This article is distributed under the terms of the Creative Commons Attribution 4.0 International License (http://creativecommons.org/licenses/by/4.0/), which permits unrestricted use, distribution, and reproduction in any medium, provided you give appropriate credit to the original author(s) and the source, provide a link to the Creative Commons license, and indicate if changes were made. The Creative Commons Public Domain Dedication waiver (http://creativecommons.org/publicdomain/zero/1.0/) applies to the data made available in this article, unless otherwise stated. 


\section{Background}

Ventilator-associated pneumonia (VAP), one form of hospital-associated pneumonia (HAP), is defined as pneumonia developing in a mechanically ventilated patient $\geq 48 \mathrm{~h}$ after tracheal intubation. When it occurs, VAP has been recognized as the leading cause of mortality among patients with nosocomial infections, and VAP patients are hospitalized on average for an additional 4-13 days with excess hospital costs [1]. Effective antimicrobial therapy requires adequate drug concentrations at the infection site. However, intravenous therapy has altered the pharmacokinetics and poor lung tissue penetration of many antimicrobial agents [2]. Thus, aerosolized antibiotic therapy directly targets airway and lung parenchyma resulting in high local concentrations and potentially higher clinical responses. Moreover, aerosolized antibiotic therapy, which directly treats lung infections, can be used to decrease systemic antibiotic doses to minimize antibiotic-associated toxicities [3].

Two previous meta-analyses reported that aerosolized antibiotic therapy might be beneficial in the treatment of VAP $[4,5]$. However, all of these studies were traditional pairwise meta-analyses comparing aerosolized antibiotics with intravenous antibiotics, and none made comparisons between the aerosolized antibiotics. A network meta-analysis (NMA), also known as mixed treatment comparison or multiple treatment comparison, is a method for concurrent comparison of multiple treatments in a single meta-analysis [6].

In the present study, our objective was to update the evidence to systematically evaluate the effect of aerosolized antibiotics in VAP patients on clinical recovery, microbiological eradication, mortality, and nephrotoxicity; we also used an NMA approach which enabled us to assess three aerosolized antibiotics by indirect comparison to determine their efficacy.

\section{Methods}

\section{Search strategy}

We included the studies from two systematic reviews $[4,5]$. In addition, we also searched the PubMed, EMBASE, and Cochrane databases from inception to September 2017 to identify potentially relevant studies. Search terms included several parameters: 1) ventilator associated pneumonia OR VAP OR respiratory infection OR respiratory tract OR hospital acquired pneumonia OR nosocomial pneumonia; 2) aerolised OR aerosolized OR inhaled OR nebulised OR nebulized. We also evaluated the reference lists of the relevant clinical trials to identify additional studies.

Studies were included if they met several criteria: 1) patients in a study population that were mechanically ventilated and diagnosed with VAP; 2) intervention which included the use of inhaled antibiotics for treatment of VAP compared with intravenous antibiotics; and 3 ) at least one of the following outcomes was reported: clinical recovery, microbiological eradication, mortality, and nephrotoxicity.

\section{Study quality assessment}

The Newcastle-Ottawa scale (NOS) for observational studies was used to assess the quality of the included studies. The NOS statement was judged on three broad perspectives (selection, comparability, and outcome) consisting of eight items. The qualities of randomized clinical trials were assessed using the risk of bias tool recommended by the Cochrane Collaboration. We assigned a value of high, unclear, or low to several parameters: 1) random sequence generation; 2) allocation concealment; 3) blinding of participants and personnel; 4) blinding of outcome assessment; 5) incomplete outcome data; 6) selective reporting; and/or 7) other bias.

\section{Statistical analysis}

We used the STATA program (Stata Corporation, College Station, Texas) for pairwise meta-analysis. The differences between the two groups were calculated as the relative risk (RR) and 95\% confidence interval (CI) for dichotomous outcomes. Heterogeneity was assessed by the Cochran $Q$ statistic and the $I^{2}$ statistic. A $P$ value $\leq 0.10$ together with an $I^{2}$ value $\geq 50 \%$ indicates significant heterogeneity. $I^{2}$ values $\leq 50 \%$ represented acceptable between-study heterogeneity, and the fixed-effects model was selected. Otherwise, the random-effects model was selected [7, 8]. Publication bias was determined using the funnel plot and assessed by Egger's test. We performed the NMA within a Bayesian framework using JAGS (version 4.2.0), R software (version 3.4.4), and the rjags and gemtc packages. The probability that each aerosolized antibiotic therapy was the best among the given treatments was determined by evaluating the rank probabilities. A higher probability of achieving rank 1 indicated a higher probability of being the best. We used these results for our interpretation.

\section{Results}

\section{Selection and characteristics of the studies}

We identified 13 studies through the reanalysis of the two systematic reviews, and four studies through the PubMed, EMBASE, and Cochrane search. These trials were published between 2000 and 2017. Accordingly, the current meta-analysis included 17 studies [9-25]. The detailed steps of the study selection process are shown in Fig. 1.

Table 1 shows the major characteristics of the included trials. There were nine observational studies and eight randomized clinical trials. Fifteen studies presented the outcome of clinical recovery $[9-21,23,24]$, and 10 presented the outcome of microbiological eradication 


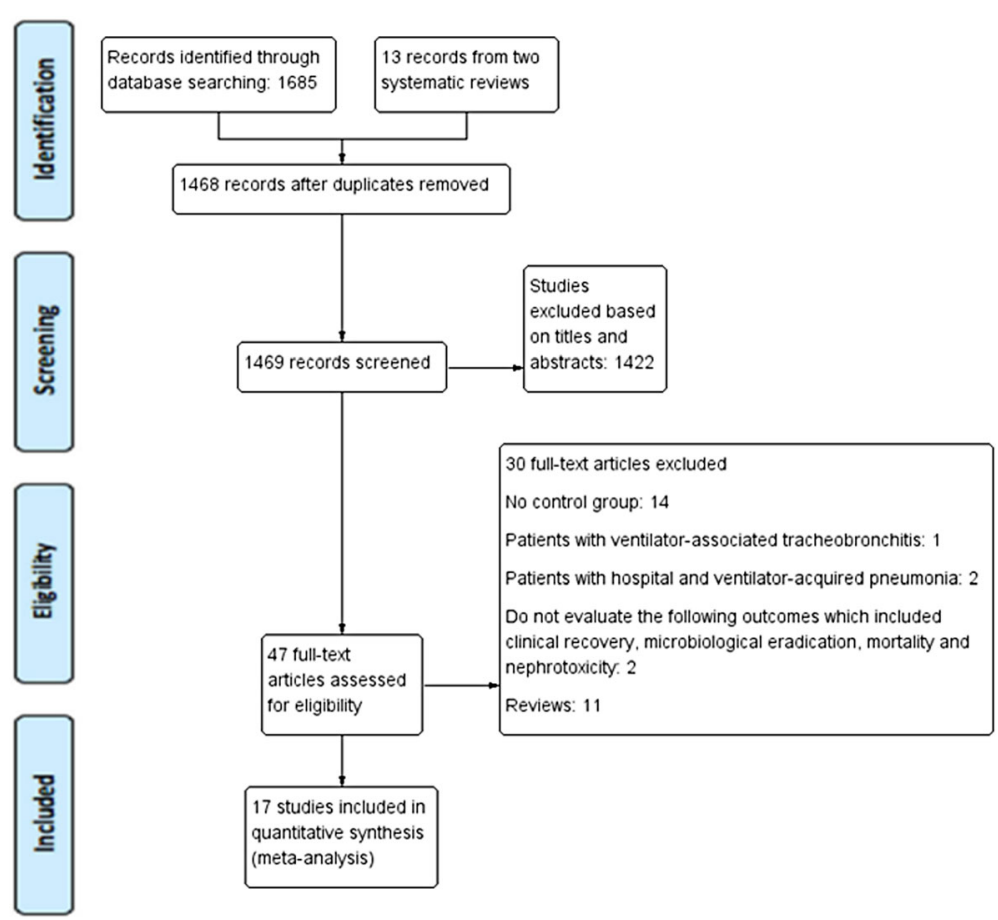

Fig. 1 Study flow diagram

$[9,10,12-15,19-22]$. Mortality was reported in 14 trials $[10,11,13-17,19-25]$, and nephrotoxicity was reported in six trials $[10,13,15,18,19,21]$. For the network meta-analysis, we excluded studies that used several inhaled antibiotics $[9,22,25]$. Three studies investigated the effect of inhaled amikacin [20, 21, 24], three studies concerned inhaled tobramycin [16-18], and eight studies concerned colistin [10-15, 19, 23].

\section{Pairwise meta-analysis}

The overall pooled RR of clinical recovery, microbiological eradication, mortality, and nephrotoxicity are shown in Additional file 1: Figure S1. Additional file 1: Figure S1A shows the estimates of clinical recovery rates from fifteen studies. Aerosolized antibiotics were associated with significantly higher rates of clinical recovery (RR 1.21, 95\% CI $1.09-1.34 ; P=0.001)$. There was no statistical heterogeneity in the fixed effect model $\left(I^{2}=36.2 \%\right)$.

Ten trials reported microbiological eradication as an outcome, and the pooled results indicated that aerosolized antibiotics also had a beneficial effect (RR 1.42 , 95\% CI $1.22-1.65 ; P<0.0001)$, despite significant heterogeneity among the studies $\left(I^{2}=68.5 \%\right)$ (Additional file 1: Figure S1B).

Mortality at the longest follow-up was available in 14 studies; aerosolized antibiotics had similar effects to control groups (RR 0.88, 95\% CI 0.74-1.04; $P=0.127$ ) with no evidence of statistical heterogeneity $\left(I^{2}=23.6 \%\right)$ (Additional file 1: Figure S1C).
Six studies presented data regarding complications on nephrotoxicity; aerosolized antibiotics were not associated with an increased risk of nephrotoxicity (RR 1.00, 95\% CI $0.72-1.39 ; P=0.995)$ with no evidence of statistical heterogeneity $\left(I^{2}=19.4 \%\right)$ (Additional file 1: Figure S1D).

\section{Publication bias}

We detected no evidence of publication bias after assessing funnel plots (Additional file 2: Figure S2) and Egger's test (clinical recovery $P=0.553$, microbiological eradication $P=0.156$, mortality $P=0.869$ and nephrotoxicity $P=0.223)$. The data suggested that there was no evidence of publication bias for clinical recovery, microbiological eradication, mortality and/or nephrotoxicity.

\section{Sensitivity analyses}

Sensitivity analyses were carried out to determine the influence of each study on the pooled RR, and the statistical findings were not materially altered by the elimination of any study (Additional file 3: Figure S3).

\section{Bayesian network meta-analysis}

There were no trials comparing outcomes among the aerosolized antibiotics, and all trials had an intravenous antibiotics arm. Thus, we pooled direct comparisons to obtain indirect comparisons by comparing aerosolized tobramycin versus aerosolized colistin, aerosolized tobramycin versus aerosolized amikacin, and aerosolized colistin versus aerosolized amikacin. In individual comparisons 


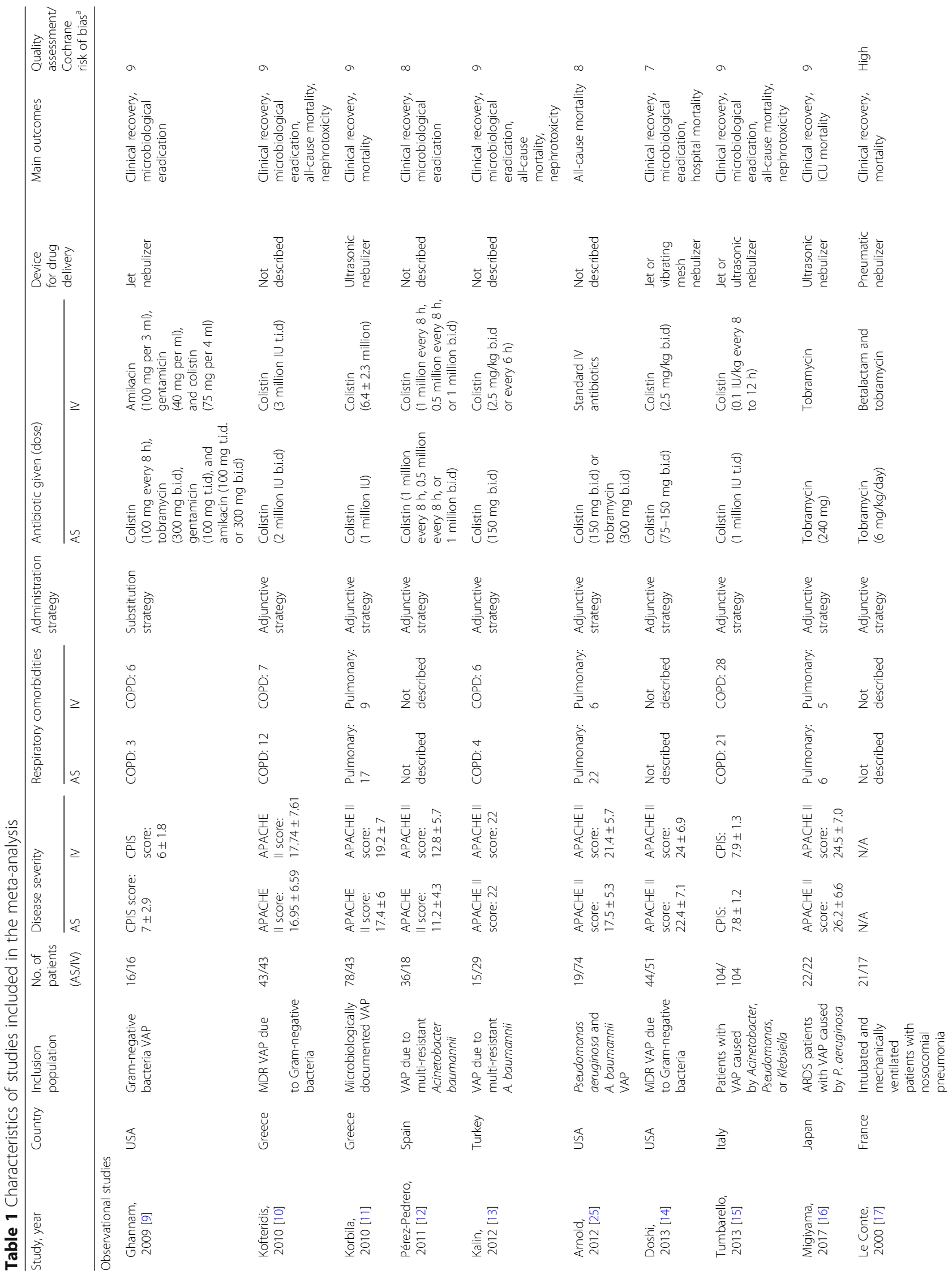




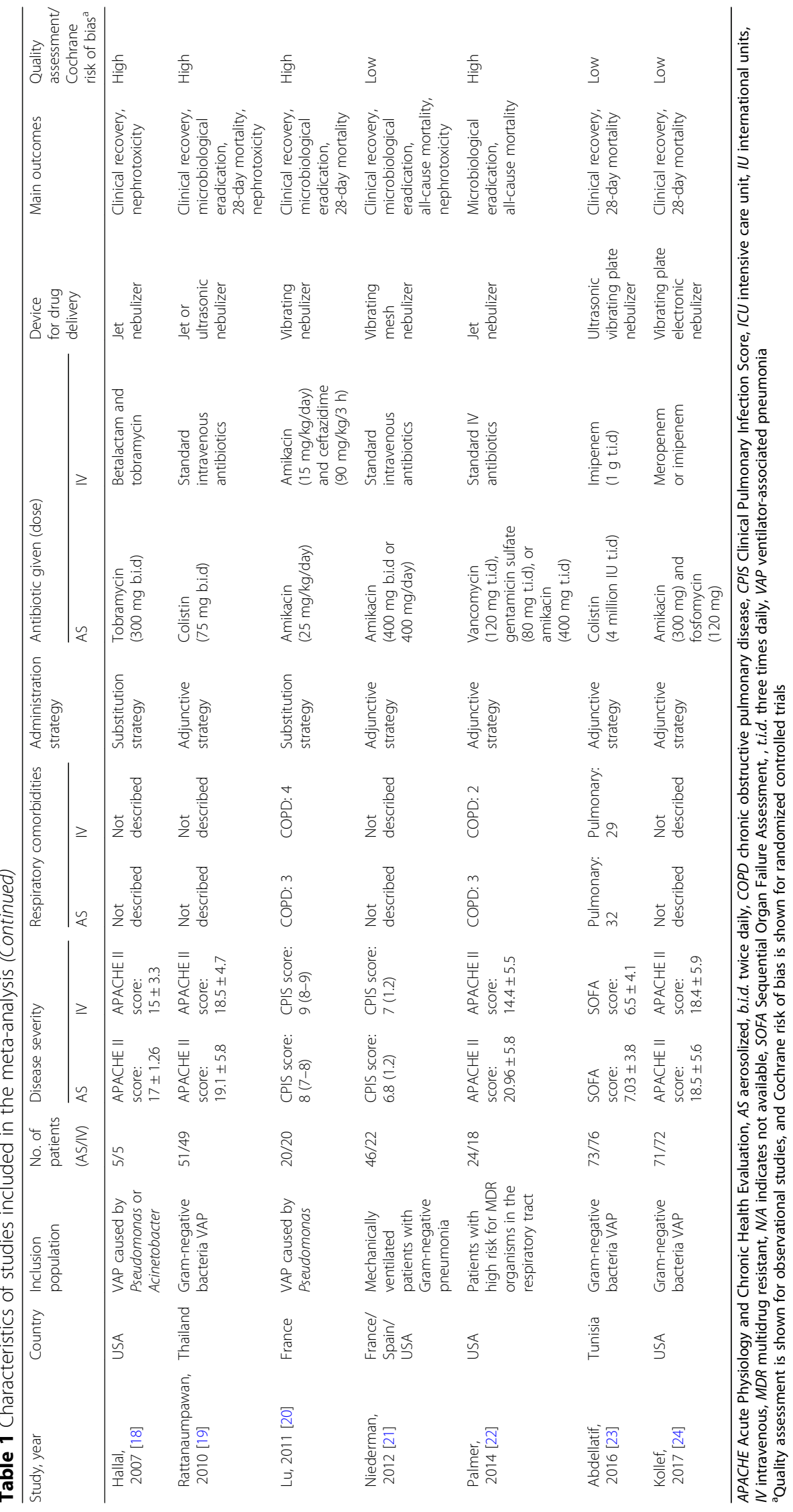


for clinical recovery using intravenous antibiotics as the reference, aerosolized tobramycin and colistin were more likely to increase the rate of clinical recovery (NMA: RR 1.7, 95\% CI 1.1-2.7; and RR 1.2, 95\% CI 1.0-1.4, respectively; Fig. 2a). Aerosolized tobramycin was associated with a significantly higher rate of clinical recovery compared with aerosolized amikacin (NMA: RR 1.8, 95\% CI 1.1-3.0; Fig. 2a). There were no significant differences among the other comparisons of the network meta-analysis for clinical recovery (Fig. 2). The assessment of rank probabilities indicated that tobramycin ( $92.3 \%$ probability) presented the greatest likelihood of improving efficacy among the evaluated aerosolized antibiotics for treating VAP, followed by colistin (SUCRA $7.4 \%$ probability) and then amikacin ( $0.2 \%$ probability) (Fig. $2 b)$. We conducted subgroup analyses according to geography, type of inhaled drug delivery system, VAP with or without multidrug resistance (MDR), type of studies, and administration strategy (Table 2). Results of our subgroup analyses from rank probabilities suggested that tobramycin displayed the best benefit for clinical recovery, followed by colistin, and then amikacin.

None of the studies used aerosolized tobramycin to evaluate the effects on microbiological eradication. Aerosolized colistin had a higher rate of microbiological eradication when compared with intravenous antibiotics (NMA: RR 1.3, 95\% CI 1.0-1.6; Fig. 3a). There were no significant differences among the other comparisons of the network meta-analysis for microbiological eradication (Fig. 3a). Ranking analysis revealed that colistin presented the highest rate of microbiological eradication (86.2\% probability) (Fig. 3b).
Taking intravenous antibiotics as the reference revealed no statistically significant differences in mortality in aerosolized amikacin and colistin, but aerosolized tobramycin showed lower mortality risk (NMA: RR 0.34, 95\% CI 0.14-0.70) (Fig. 4a). Aerosolized tobramycin was also associated with significantly lower mortality compared with aerosolized amikacin (NMA: RR 0.26, 95\% CI 0.089-0.67) and aerosolized colistin (NMA: RR 0.39, 95\% CI 0.16-0.84) (Fig. 4a). Ranking analysis revealed that the hierarchy for efficacy in avoiding death (highest to lowest rank) was tobramycin ( $98.5 \%$ probability), followed by colistin $(0.99 \%$ probability) and then amikacin $(0.37 \%$ probability) (Fig. 4b). Table 2 shows the rank probabilities of subgroup analyses that provided the hierarchies for the mortality of the aerosolized antibiotics. Ranking analysis revealed that tobramycin was the best treatment in terms of reducing hospital mortality.

The forest plot for the risk of nephrotoxicity is shown in Fig. 5a. There were no significant differences among any comparisons of the network meta-analysis (Fig. 5a). Ranking analysis revealed that the hierarchy for safety in avoiding renal toxicity (highest to lowest rank) was tobramycin (69.4\% probability), followed by amikacin (29.4\% probability) and then colistin ( $0.5 \%$ probability) (Fig. 5 b).

\section{Discussion}

Previous studies have demonstrated that aerosolized antibiotics affected the clinical prognosis in VAP treatment. Whether aerosolized antibiotics are beneficial or harmful for critically ill patients has long been a matter of debate. In this meta-analysis, we found that aerosolized antibiotics

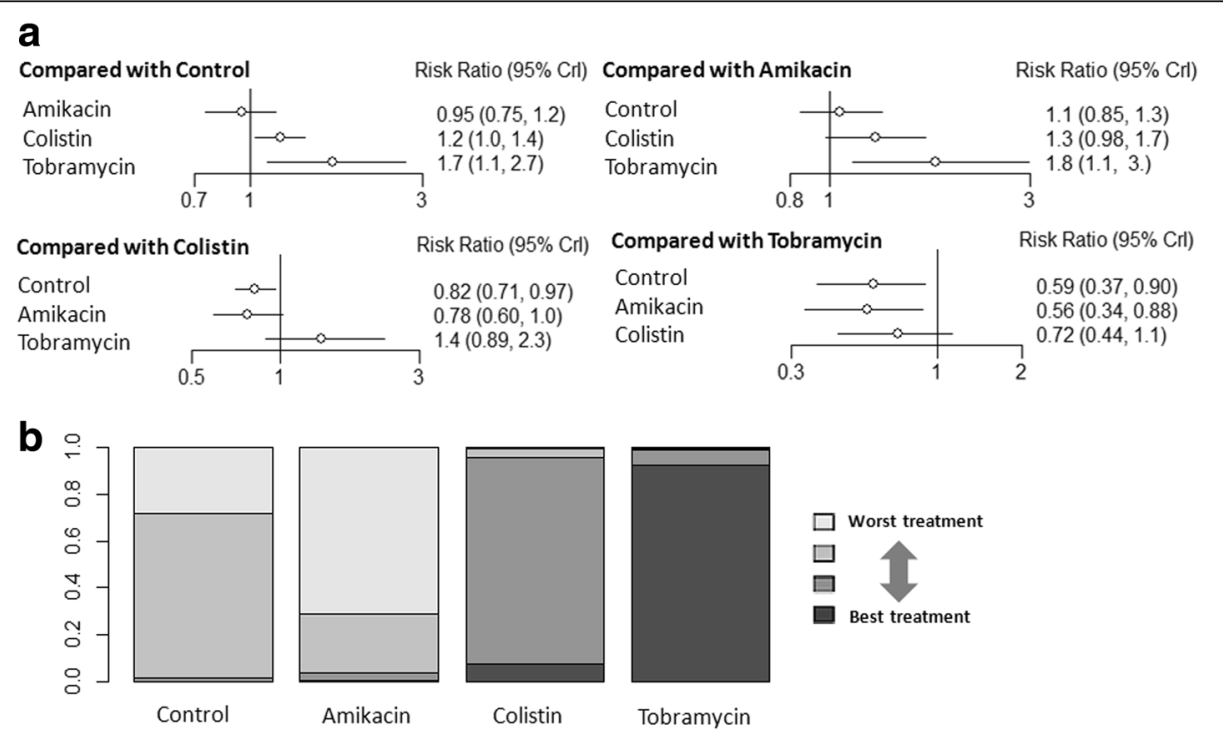

Fig. 2 a Network estimates among aerosolized antibiotics for clinical recovery. b Rank probabilities among aerosolized antibiotics for clinical recovery based on the network meta-analysis. The number of patients in each antibiotic arm: control, 476; amikacin, 135; colistin, 372; and tobramycin, 48. Cl confidence interval 


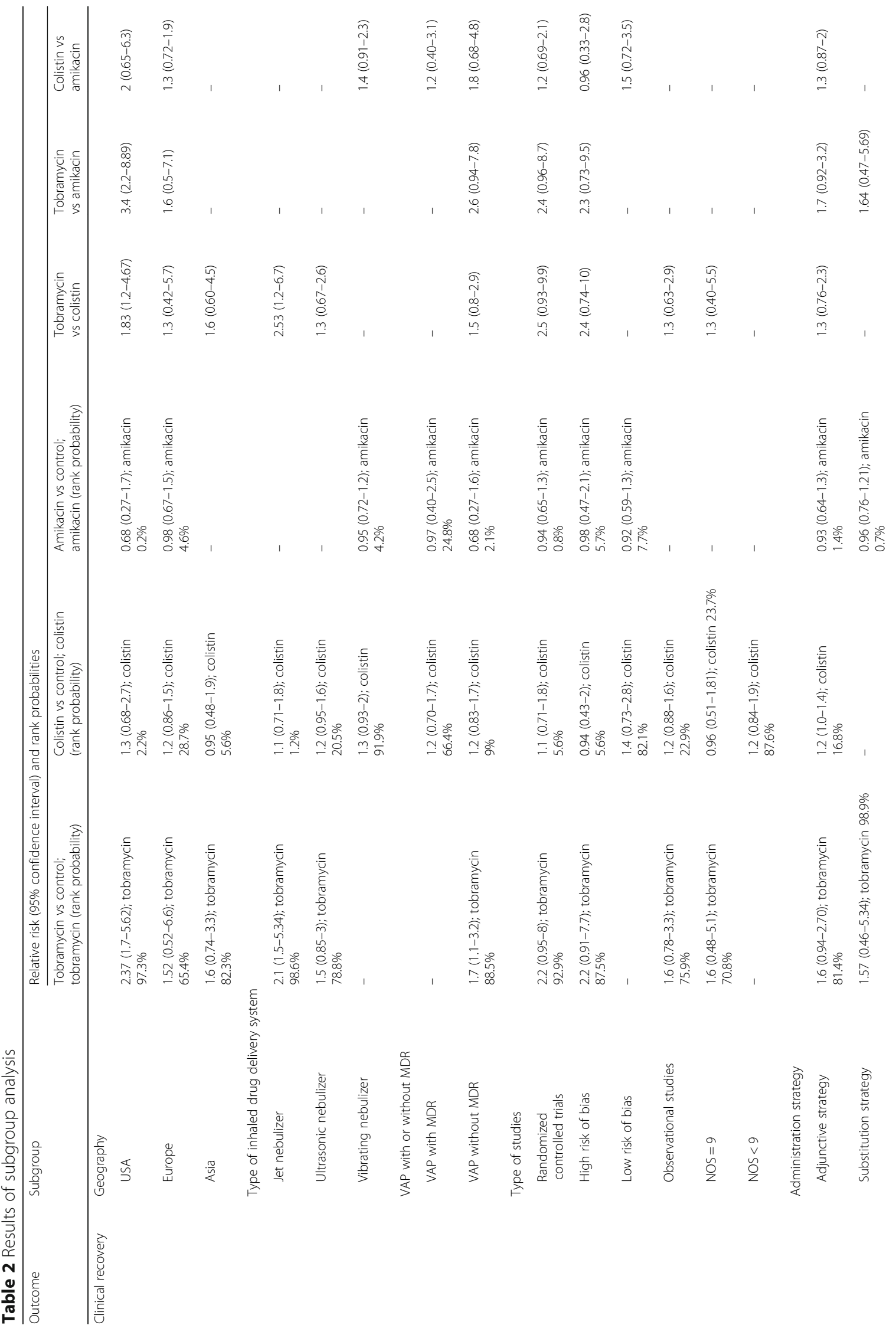




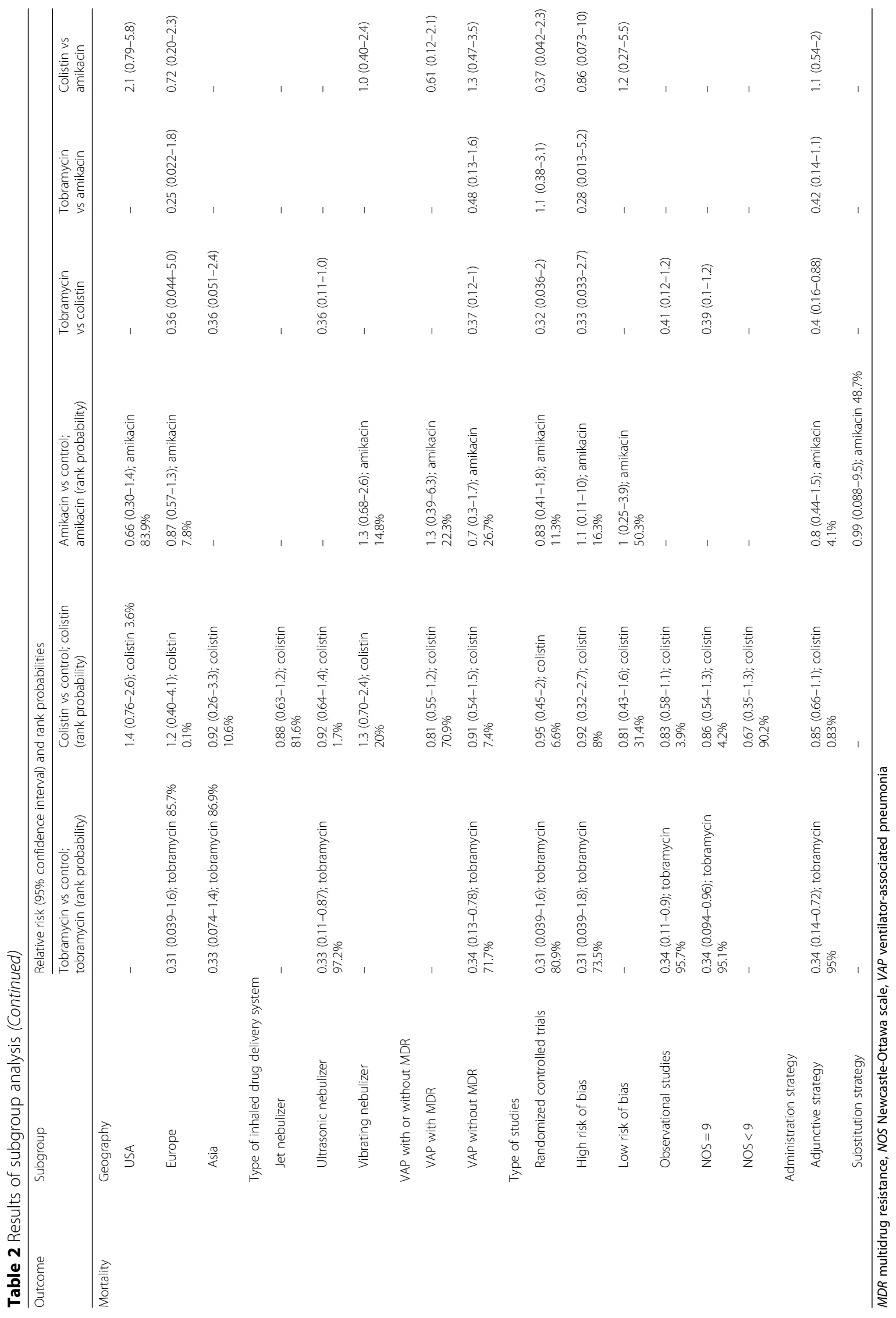



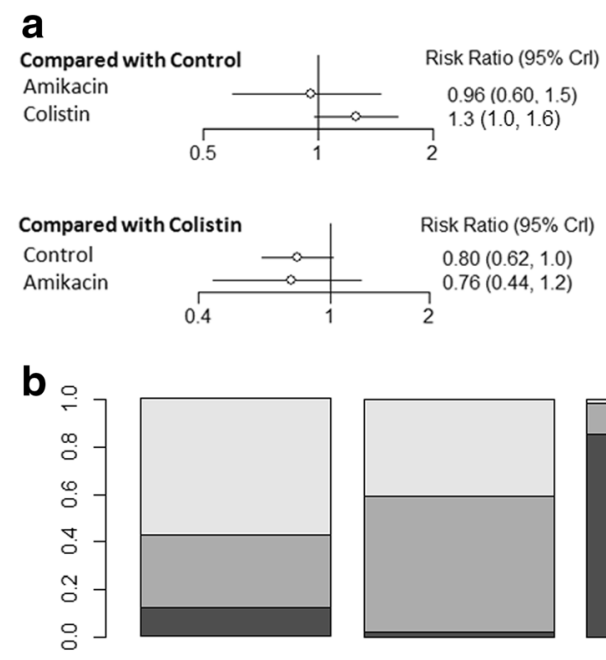

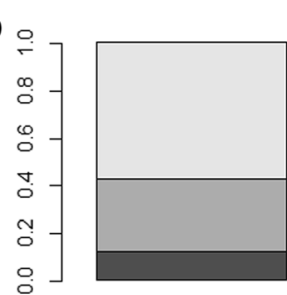

Control

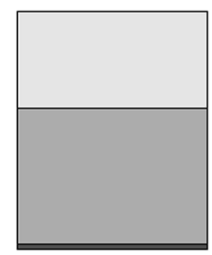

Amikacin

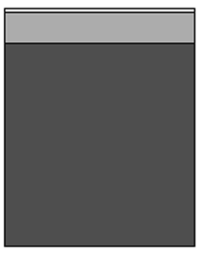

Colistin

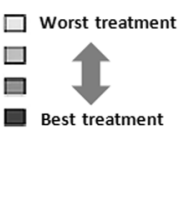

Fig. 3 a Network estimates among aerosolized antibiotics for microbiological eradication. b Rank probabilities among aerosolized antibiotics for microbiological eradication based on the network meta-analysis. The number of patients in each antibiotic arm: control, 271; amikacin, 74; and colistin, 241. Cl confidence interval

could improve clinical recovery and microbiological eradication. In addition, there were no differences in terms of outcomes such as mortality or nephrotoxicity. The present network meta-analysis and ranking analysis suggest that the probability of being the best aerosolized therapy for VAP was tobramycin with respect to clinical recovery and mortality, and colistin for microbiological eradication. Although this was a hypothesis-generating study, the current study is the first network meta-analysis to compare different aerosolized antibiotics in the treatment of VAP. The present meta-analysis is different from the two previous ones $[4,5]$. We have updated the studies for this analysis, separately compared each aerosolized antibiotic with intravenous therapy, assessed the quality of evidence, detected publication bias, and conducted sensitivity analyses. A previous meta-analysis by Zampieri et al. investigated the role of aerosolized antibiotics in VAP treatment [4]. This analysis found the same benefits as our analysis on clinical recovery, mortality, and nephrotoxicity, but aerosolized antibiotics did not increase the rate for microbiological eradication. Contrary to their microbiological eradication findings, our analysis indicated that

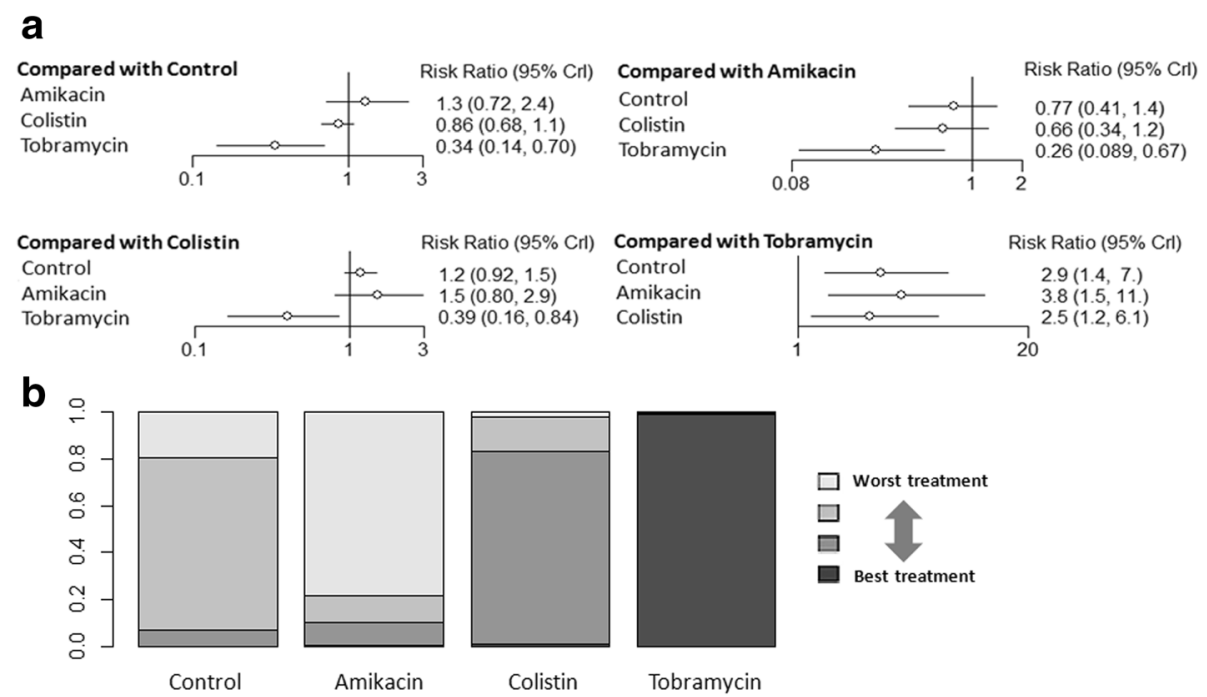

Fig. 4 a Network estimates among aerosolized antibiotics for mortality. b Rank probabilities among aerosolized antibiotics for mortality based on the network meta-analysis. The number of patients in each antibiotic arm: control, 464; amikacin, 138; colistin, 362; and tobramycin, 43. Cl confidence interval 

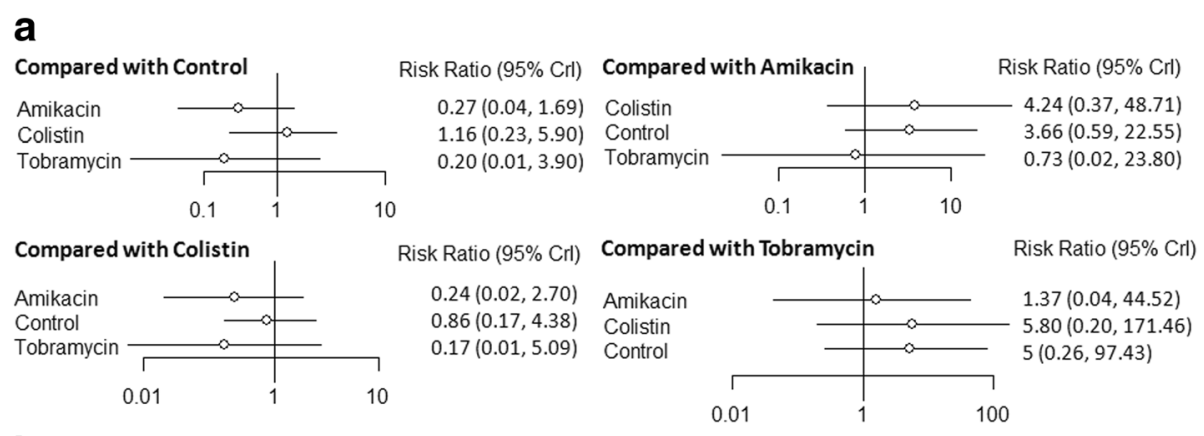

b
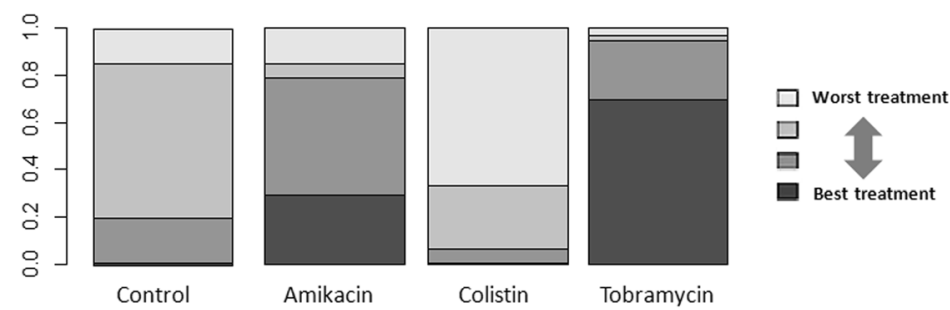

Fig. 5 a Network estimates among aerosolized antibiotics for nephrotoxicity. b Rank probabilities among aerosolized antibiotics for nephrotoxicity based on the network meta-analysis. The number of patients in each antibiotic arm: control, 231; amikacin, 45; colistin, 5; and tobramycin, 230. Cl confidence interval

aerosolized antibiotics also have a beneficial effect. Thus, there may have been an inadequate sample size to correct the type II error in the study of Zampieri et al. In addition, we used network meta-analysis to identify an optimal aerosolized antibiotic for VAP.

VAP remains an important infectious complication of mechanical ventilation and is commonly due to MDR pathogens (Enterococcus faecium, Staphylococcus aureus, Klebsiella pneumoniae, Acinetobacter baumannii, P. aeruginosa, and enterobacter spp.). Because of MDR pathogens, the capability of commonly used intravenous antibiotics to cross the lung parenchyma is significantly inhibited [26, 27]. Inadequate concentrations of intravenous antibiotics may result in failure to reach inhibitory concentrations. Aerosolized antibiotics may be a useful treatment since aerosolization directly targets the airways and localizes the drug to the pulmonary parenchyma, thus bypassing the poor lung penetration of many antibiotics. Aerosolized antibiotics therefore make it possible to achieve high local antibiotic concentrations relative to an organism's minimum inhibitory concentration while concurrently minimizing systemic toxicities by concentrating antibiotics in the lungs rather than spreading them throughout the body [28].

The appropriate aerosolized antibiotic remains a matter of controversy, and the most studied are colistin and aminoglycosides. The selection of the aerosolized antibiotic should encompass several properties and characteristics to achieve maximum effectiveness including: 1 ) activity against the causative pathogen; 2) physical properties to ensure maximal pulmonary delivery and minimal extrapulmonary loss; and 3) the achievement of adequate concentrations in the lung well above the pathogen's minimum inhibitory concentration, taking into account the need for the prevention of resistance and the presence of biofilm [29].

In the presence of severe experimental lung infection, aminoglycoside plasma concentrations were found in the same range after nebulization and intravenous administration [30]. Furthermore, inhaled aminoglycosides prevent growth of bacterial biofilms [31]. Inhaled tobramycin has a wide range of activity against Gram-negative organisms, including $P$. aeruginosa. Tobramycin is also preferable for inhaled use because of moderately lipophilic and positively charged small molecules [27]. Amikacin is another aminoglycoside. The low bioavailability of nebulized amikacin may result in administration of high dosesand hence potentially increase systemic toxicities. Mohr et al. reported that pharmacokinetic properties and dosing strategies were better defined for inhaled tobramycin than for inhaled amikacin [32]. Studies in animals have shown that aerosolized colistin has a limited systemic diffusion in pneumonia [33]. Likewise, inhaled colistin also has high mucin binding that might lead to insufficient antibiotic effectiveness to kill bacteria [34]. As a consequence, a dosage exceeding the common dose of colistin treating VAP may enhance the rate of clinical recovery and microbiological eradication but increase the risk of toxicity. Therefore, tobramycin may have more potential effects on clinical recovery and be promising for its effects on mortality and nephrotoxicity.

In seeking to optimize treatment, network meta-analysis was used to gain further insight into the best 
preferred agent. The present network meta-analysis found that aerosolized tobramycin and colistin (especially tobramycin) were more likely to increase the rate of clinical recovery. Aerosolized tobramycin also had additional benefits on mortality, and aerosolized colistin had a higher rate of microbiological eradication. Amikacin had no significant differences in clinical recovery, microbiological eradication, mortality, or nephrotoxicity compared with intravenous antibiotics. Accordingly, the present network meta-analysis and the clustered ranking plot suggest that tobramycin present the best outcome for clinical recovery and mortality, and colistin present the best outcome for microbiological eradication.

Aerosolized antibiotics seem to be attractive alternatives for VAP treatment. However, an in-vivo randomized controlled trial examining adjuvant therapy with aerosolized antibiotics in VAP with varying degrees of bacterial resistance has shown that aerosolized antibiotics have no effect on the clinical course, including the serial Clinical Pulmonary Infection Score (CPIS), clinical cure rates, ventilator-free and ICU days, or mortality [24]. Despite the weak evidence in our analysis suggesting that the administration of aerosolized antibiotics such as colistin or tobramycin instead of the administration of intravenous antibiotics might be a good option for the treatment of VAP, there may as yet be no recommendations for using them in standard clinical practice. The rationale for the recommendation of aerosolized antibiotics requires other large multicenter trials to determine if these preliminary findings will result in better clinical activity and decreased microbial resistance in patients with VAP.

Some important limitations of the study should not be overlooked. First, different criteria for clinical recovery, microbiological eradication, and nephrotoxicity in the included trials might affect the robustness of our findings. The endpoint of microbiologic eradication is of uncertain value when measured during the use of inhaled antibiotics since the inhaled antibiotics are in the respiratory sample sent for culture which may influence the accuracy of the endpoint. Second, there are many factors which may impact the effect of inhaled antibiotics including types of nebulizers, ventilator settings, ventilator and circuit connections, and circuit humidification and filtering. We could not obtain sufficient data suggesting that other study design issues should be addressed. Third, the eligible studies had substantial differences in terms of the causative microorganisms, and the impact of inhaled antibiotics might vary against different pathogens. Fourth, a major concern when small studies are included in our analysis is the presence of a "smallstudy effect", which arises from publication bias and selection bias and results in lower methodological quality [35]. The small-study effect was present in the analysis of treatment success in VAP patients with respect to aerosolized antibiotics. This should be taken into careful consideration when evaluating the results of the present analysis. Fifth, although network meta-analysis allowed us to compare the efficacy and safety of inhaled antibiotics, we acknowledge the limitation that interpretation of a network meta-analysis relies on the insufficient indirect comparison of outcomes through common comparators. Sixth, we included observational studies and randomized controlled trials; however, observational studies have the risk of overrated pooled estimates.

\section{Conclusions}

Aerosolized antibiotics appear to be useful treatment for VAP with respect to clinical recovery and microbiological eradication, and do not seem to increase mortality or nephrotoxicity risks. Our network meta-analysis in patients with VAP suggests that clinical recovery benefits are associated with aerosolized tobramycin and colistin (especially tobramycin), microbiological eradication with aerosolized colistin, and survival with aerosolized tobramycin, mostly based on observational studies. Moreover, we should take into account that some studies using aerosolized antibiotics failed to show an improvement, and data of other studies are still not published. Due to the low level of evidence, definitive recommendations cannot be made before additional large randomized studies are carried out.

\section{Key messages}

- Aerosolized antibiotics appear to be beneficial for the treatment of VAP.

- The current evidence from network meta-analysis indicates that aerosolized tobramycin is associated with the best outcome on clinical recovery and mortality.

- The current evidence from network meta-analysis indicates that aerosolized colistin is associated with improved clinical recovery and microbiological eradication.

\section{Additional files}

Additional file 1: Figure S1. Forest plots showing the effect of aerosolized antibiotics on (A) clinical recovery, (B) microbiological eradication, (C) mortality, and (D) nephrotoxicity. (TIF 8222 kb)

Additional file 2: Figure S2. Funnel plots for the rate of $(A)$ clinical recovery, (B) microbiological eradication, (C) mortality, and (D) nephrotoxicity. (TIF $2106 \mathrm{~kb}$ )

Additional file 3: Figure S3. Sensitivity analyses of the included studies reporting aerosolized antibiotics on (A) clinical recovery, (B) microbiological eradication, (C) mortality, and (D) nephrotoxicity. (TIF 4998 kb)

\section{Abbreviations}

$\mathrm{Cl}$ : Confidence interval; $\mathrm{Cl}$ : Confidence interval; HAP: Hospital-associated pneumonia; HAP: Hospital-associated pneumonia; MDR: Multidrug resistant; 
NMA: Network meta-analysis; NMA: Network meta-analysis; NOS: NewcastleOttawa scale; NOS: Newcastle-Ottawa scale; RCT: Randomized controlled trials; RR: Risk ratio; RR: Risk ratio; VAP: Ventilator-associated pneumonia; VAP: Ventilator-associated pneumonia

\section{Funding}

This work was supported by Major Projects of the National Natural Science Foundation of China (81490532 to HHS and 91642202 to WL), and the program for Key Site of National Clinical Research Center for Respiratory Disease.

\section{Availability of data and materials}

All data generated and/or analyzed during this study are included in this published article.

\section{Authors' contributions}

FX and LLH conducted the literature searches, selected the studies, and assessed the study quality. FX, LLH, and LQC screened the abstracts, selected the studies meeting the inclusion criteria, extracted the data, and assessed the study quality. FX and LLH drafted and revised the manuscript. All authors read and approved the final manuscript.

\section{Ethics approval and consent to participate}

Not applicable.

\section{Consent for publication}

Not applicable.

\section{Competing interests}

The authors declare that they have no competing interests.

\section{Publisher's Note}

Springer Nature remains neutral with regard to jurisdictional claims in published maps and institutional affiliations.

Received: 22 January 2018 Accepted: 22 June 2018

Published online: 15 November 2018

\section{References}

1. Kollef $\mathrm{MH}$. What is ventilator-associated pneumonia and why is it important? Respir Care. 2005;50(6):714-21. discussion 721-714

2. Ehrmann S, Chastre J, Diot P, Lu Q. Nebulized antibiotics in mechanically ventilated patients: a challenge for translational research from technology to clinical care. Ann Intensive Care. 2017;7(1):78.

3. Palmer LB. Aerosolized antibiotics in critically ill ventilated patients. Curr Opin Crit Care. 2009;15(5):413-8.

4. Zampieri FG, Nassar AP Jr, Gusmao-Flores D, Taniguchi LU, Torres A, Ranzani OT. Nebulized antibiotics for ventilator-associated pneumonia: a systematic review and meta-analysis. Critical Care. 2015;19:150.

5. Sole-Lleonart C, Rouby JJ, Blot S, Poulakou G, Chastre J, Palmer LB, Bassetti M, Luyt CE, Pereira JM, Riera J, et al. Nebulization of antiinfective agents in invasively mechanically ventilated adults: a systematic review and meta-analysis. Anesthesiology. 2017;126(5):890-908.

6. Naing C, Reid SA, Aung K. Comparing antibiotic treatment for leptospirosis using network meta-analysis: a tutorial. BMC Infect Dis. 2017;17(1):29.

7. Jiang $P, L \vee Q, L a i T, X u F$. Does hypomagnesemia impact on the outcome of patients admitted to the intensive care unit? A systematic review and meta-analysis. Shock. 2017;47(3):288-95.

8. Du F, Jiang P, He S, Song D, Xu F. Antiplatelet therapy for critically ill patients: a pairwise and Bayesian network meta-analysis. Shock. 2018;49(6):616-24.

9. Ghannam DE, Rodriguez GH, Raad II, Safdar A. Inhaled aminoglycosides in cancer patients with ventilator-associated gram-negative bacterial pneumonia: safety and feasibility in the era of escalating drug resistance. Eur J Clin Microbiol Infect Dis. 2009:28(3):253-9.

10. Kofteridis DP, Alexopoulou C, Valachis A, Maraki S, Dimopoulou D, Georgopoulos D, Samonis G. Aerosolized plus intravenous colistin versus intravenous colistin alone for the treatment of ventilator-associated pneumonia: a matched case-control study. Clin Infect Dis. 2010;51(11):1238-44.

11. Korbila IP, Michalopoulos A, Rafailidis PI, Nikita D, Samonis G, Falagas ME. Inhaled colistin as adjunctive therapy to intravenous colistin for the treatment of microbiologically documented ventilator-associated pneumonia: a comparative cohort study. Clin Microbiol Infect. 2010;16(8):1230-6.

12. Perez-Pedrero MJ, Sanchez-Casado M, Rodriguez-Villar S. Nebulized colistin treatment of multi-resistant Acinetobacter baumannii pulmonary infection in critical ill patients. Med Int. 2011;35(4):226-31.

13. Kalin G, Alp E, Coskun R, Demiraslan H, Gundogan K, Doganay M. Use of high-dose IV and aerosolized colistin for the treatment of multidrug-resistant Acinetobacter baumannii ventilator-associated pneumonia: do we really need this treatment? J Infect Chemother. 2012;18(6):872-7.

14. Doshi NM, Cook CH, Mount KL, Stawicki SP, Frazee EN, Personett HA, Schramm GE, Arnold HM, Murphy CV. Adjunctive aerosolized colistin for multi-drug resistant gram-negative pneumonia in the critically ill: a retrospective study. BMC Anesthesiol. 2013;13(1):45.

15. Tumbarello M, De Pascale G, Trecarichi EM, De Martino S, Bello G, Maviglia R, Spanu T, Antonelli M. Effect of aerosolized colistin as adjunctive treatment on the outcomes of microbiologically documented ventilator-associated pneumonia caused by colistin-only susceptible gram-negative bacteria. Chest. 2013;144(6):1768-75.

16. Migiyama Y, Hirosako S, Tokunaga K, Migiyama E, Tashiro T, Sagishima K, Kamohara H, Kinoshita $Y$, Kohrogi $H$. Aerosolized tobramycin for Pseudomonas aeruginosa ventilator-associated pneumonia in patients with acute respiratory distress syndrome. Pulm Pharmacol Ther. 2017;45:142-7.

17. Le Conte P, Potel G, Clementi E, Legras A, Villers D, Bironneau E, Cousson J, Baron D. Administration of tobramycin aerosols in patients with nosocomial pneumonia: a preliminary study. Presse Med. 2000;29(2):76-8.

18. Hallal A, Cohn SM, Namias N, Habib F, Baracco G, Manning RJ, Crookes B, Schulman $\mathrm{Cl}$. Aerosolized tobramycin in the treatment of ventilator-associated pneumonia: a pilot study. Surg Infect. 2007;8(1):73-82.

19. Rattanaumpawan $P$, Lorsutthitham J, Ungprasert P, Angkasekwinai N, Thamlikitkul V. Randomized controlled trial of nebulized colistimethate sodium as adjunctive therapy of ventilator-associated pneumonia caused by gram-negative bacteria. J Antimicrob Chemother. 2010;65(12):2645-9.

20. Lu Q, Yang J, Liu Z, Gutierrez C, Aymard G, Rouby JJ. Nebulized ceftazidime and amikacin in ventilator-associated pneumonia caused by Pseudomonas aeruginosa. Am J Respir Crit Care Med. 2011;184(1):106-15.

21. Niederman MS, Chastre J, Corkery K, Fink JB, Luyt CE, Garcia MS. BAY41-6551 achieves bactericidal tracheal aspirate amikacin concentrations in mechanically ventilated patients with gram-negative pneumonia. Intensive Care Med. 2012;38(2):263-71.

22. Palmer LB, Smaldone GC. Reduction of bacterial resistance with inhaled antibiotics in the intensive care unit. Am J Respir Crit Care Med. 2014;189(10):1225-33.

23. Abdellatif S, Trifi A, Daly F, Mahjoub K, Nasri R, Ben Lakhal S. Efficacy and toxicity of aerosolised colistin in ventilator-associated pneumonia: a prospective, randomised trial. Ann Intensive Care. 2016;6(1):26.

24. Kollef MH, Ricard JD, Roux D, Francois B, Ischaki E, Rozgonyi Z, Boulain T, Ivanyi Z, Janos G, Garot D, et al. A randomized trial of the amikacin Fosfomycin inhalation system for the adjunctive therapy of gram-negative ventilator-associated pneumonia: IASIS trial. Chest. 2017;151(6):1239-46.

25. Arnold HM, Sawyer AM, Kollef MH. Use of adjunctive aerosolized antimicrobial therapy in the treatment of Pseudomonas aeruginosa and Acinetobacter baumannii ventilator-associated pneumonia. Respir Care. 2012;57(8):1226-33.

26. Imberti R, Cusato M, Villani P, Carnevale L, lotti GA, Langer M, Regazzi M. Steady-state pharmacokinetics and BAL concentration of colistin in critically ill patients after IV colistin methanesulfonate administration. Chest. 2010; 138(6):1333-9.

27. Grgurich PE, Hudcova J, Lei Y, Sarwar A, Craven DE. Management and prevention of ventilator-associated pneumonia caused by multidrug-resistant pathogens. Expert Rev Respir Med. 2012;6(5):533-55.

28. Zhang C, Berra L, Klompas M. Should aerosolized antibiotics be used to treat ventilator-associated pneumonia? Respir Care. 2016;61(6):737-48.

29. Poulakou G, Matthaiou DK, Nicolau DP, Siakallis G, Dimopoulos G. Inhaled antimicrobials for ventilator-associated pneumonia: practical aspects. Drugs. 2017;77(13):1399-412.

30. Elman M, Goldstein I, Marquette $\mathrm{CH}$, Wallet F, Lenaour G, Rouby JJ. Influence of lung aeration on pulmonary concentrations of nebulized and intravenous amikacin in ventilated piglets with severe bronchopneumonia. Anesthesiology. 2002;97(1):199-206.

31. Adair CG, Gorman SP, Byers LM, Jones DS, Feron B, Crowe M, Webb HC, McCarthy GJ, Milligan KR. Eradication of endotracheal tube biofilm by nebulised gentamicin. Intensive Care Med. 2002;28(4):426-31. 
32. Mohr AM, Sifri ZC, Horng HS, Sadek R, Savetamal A, Hauser CJ, Livingston DH. Use of aerosolized aminoglycosides in the treatment of gram-negative ventilator-associated pneumonia. Surg Infect. 2007;8(3):349-57.

33. Pereira GH, Muller PR, Levin AS. Salvage treatment of pneumonia and initial treatment of tracheobronchitis caused by multidrug-resistant gram-negative bacilli with inhaled polymyxin B. Diagn Microbiol Infect Dis. 2007;58(2):235-40.

34. Huang JX, Blaskovich MA, Pelingon R, Ramu S, Kavanagh A, Elliott AG, Butler MS, Montgomery AB, Cooper MA. Mucin binding reduces colistin antimicrobial activity. Antimicrob Agents Chemother. 2015;59(10):5925-31.

35. Sterne JA, Gavaghan D, Egger M. Publication and related bias in meta-analysis: power of statistical tests and prevalence in the literature. J Clin Epidemiol. 2000;53(11):1119-29. 